\title{
Mit geschärftem Blick und spitzer Feder Rätseln der menschlichen Seele auf der Spur
}

Isabel Zwyssig

Korrespondenz: Isabel Zwyssig Länggassstrasse 75 CH-3012 Bern students.unibe.ch
Schneidend kalt weht an jenem Nachmittag im Februar die Bise durch Thun. Von dichten, grauen Wolken verhangen, erweckt der Himmel den Anschein, als müsste es jeden Moment anfangen zu regnen. In der Tat verlockt die trübe Wetterlage zum Gedanken, sich mit einem spannenden Buch auf das Sofa zurückzuziehen. «Spannendes Buch» ist auch das Stichwort zu meinem Einsatz. Ich bin auf dem Weg zu Esther Pauchard, Fachärztin für Psychiatrie und Psychotherapie sowie Autorin der beiden Kriminalromane «Jenseits der Couch» (2010) und «Jenseits der Mauern» (2012) - beide erschienen im Berner Nydegg Verlag. Wenig später sitzen wir im holzvertäfelten Esszimmer, wo Esther Pauchard zu erzählen beginnt. «Als ich mich ein halbes Jahr nach der Geburt meiner zweiten Tochter dazu entschlossen hatte, einen Krimi zu schreiben, ging es mir zunächst vor allem darum, etwas Neues auszuprobieren.» So viel Unternehmungslust wirkt erstaunlich - vor allem, wenn man bedenkt, wie verantwortungsvoll und vielseitig die Aufgaben sind, welche die Mutter von zwei Töchtern im Alter von vier und sechs Jahren Tag für Tag erfüllt. Neben ihrem 24-Stunden-Job als Mama ist sie nämlich noch als Oberärztin in der Suchtfachklinik Selhofen bei Burgdorf sowie in einer privaten Praxis in Thun tätig. «Gerade weil ich es gewohnt bin, anspruchsvollen Verpflichtungen nachzugehen und andere zu führen, stellte das Buchprojekt eine willkommene, interessante Abwechslung zur Routine des Alltags dar. Vielleicht wirkt Schreiben sogar als MidlifeCrisis-Prophylaxe.» In der ruhigen, klaren Altstimme schwingt leichte Belustigung mit. Freute sich Esther Pauchard während ihrer Schulzeit besonders am Verfassen von Aufsätzen, so sind heute ihre Verlaufsberichte sehr ausführlich und wohlformuliert gehalten. «Ob dieser Macke ist schon so manche Sekretärin beinahe verzweifelt», schmunzelt sie. Zweifellos hat die 40-Jährige immer gerne geschrieben, und auch Krimis faszinieren sie seit langem. Dies dürfte nicht zuletzt damit zu tun haben, dass Esther Pauchard durch ihre berufliche Tätigkeit oftmals mit Geschichten konfrontiert wird, die kriminelles Potential bergen. Gerade bei schizophrenen Patienten beispielsweise komme es regelmässig vor, dass schwerwiegende Anschuldigungen gegen Angehörige oder den Staat ausgesprochen würden, so die Fachfrau. Im Normalfall erweisen sich solche Vorwürfe zum Glück als haltlos. Wenn man jedoch ein

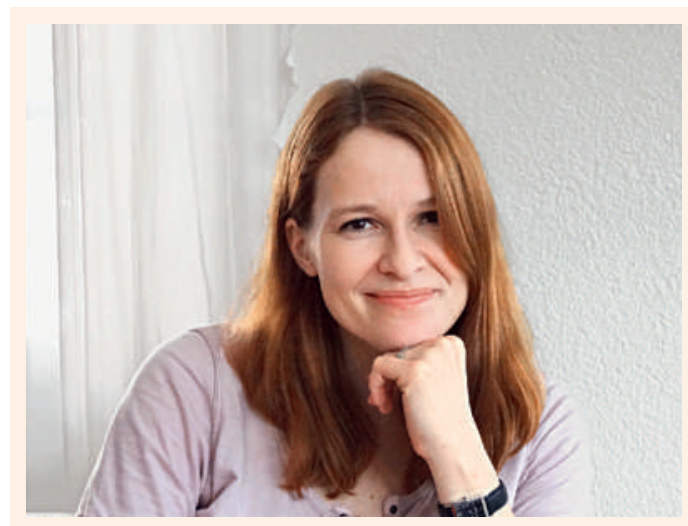

\section{Zur Person}

Esther Pauchard, geboren 1973, hat in Bern Medizin studiert. Sie ist heute Oberärztin an der Suchtfachklinik Selhofen bei Burgdorf und in einer privaten Praxis in Thun tätig. Die Mutter von zwei Töchtern im Alter von vier und sechs Jahren ist mit einem Hausarzt verheiratet und lebt mit ihrer Familie in Thun.

so überzeugter Agatha-Christie-Fan ist wie Esther Pauchard, dann passiert es durchaus, dass die Fantasie bisweilen mit einem durchgeht. Was also liegt angesichts dieser Konstellation näher, als sich selbst als Krimiautorin zu versuchen? Mitnichten haben dabei einzig die persönlichen Neigungen der Psychiaterin eine Rolle gespielt. Vielmehr waren eine unbefriedigende Lektüre und ihr gelegentlich impulsives Wesen ausschlaggebend für den Startschuss zum eigenen Buchprojekt: «Aufgebracht wie ich damals war, habe ich beschlossen, die Sache selber in die Hand zu nehmen. Schliesslich, so scheint es, kann ja jeder einen Krimi aufs Papier bringen.»

\section{Einblick nehmen in eine fremde Welt}

Und tatsächlich: Innerhalb von rekordverdächtigen drei Monaten hatte Esther Pauchard ihren Erstling niedergeschrieben. Dank einem sorgfältig ausgearbeiteten Konzept und dem Bewusstsein, dass sie schreiben darf, aber nicht muss, sind Kreativitätsblockaden ausgeblieben. Aus Jux hat sie ihr Manuskript an verschiedene Verlage geschickt. Nachdem sie vom Nydegg Verlag einen positiven Bescheid erhalten hatte, musste die sonst so schlagfertige Ärztin erst einmal leer schlucken. «Mit einer Publikation 


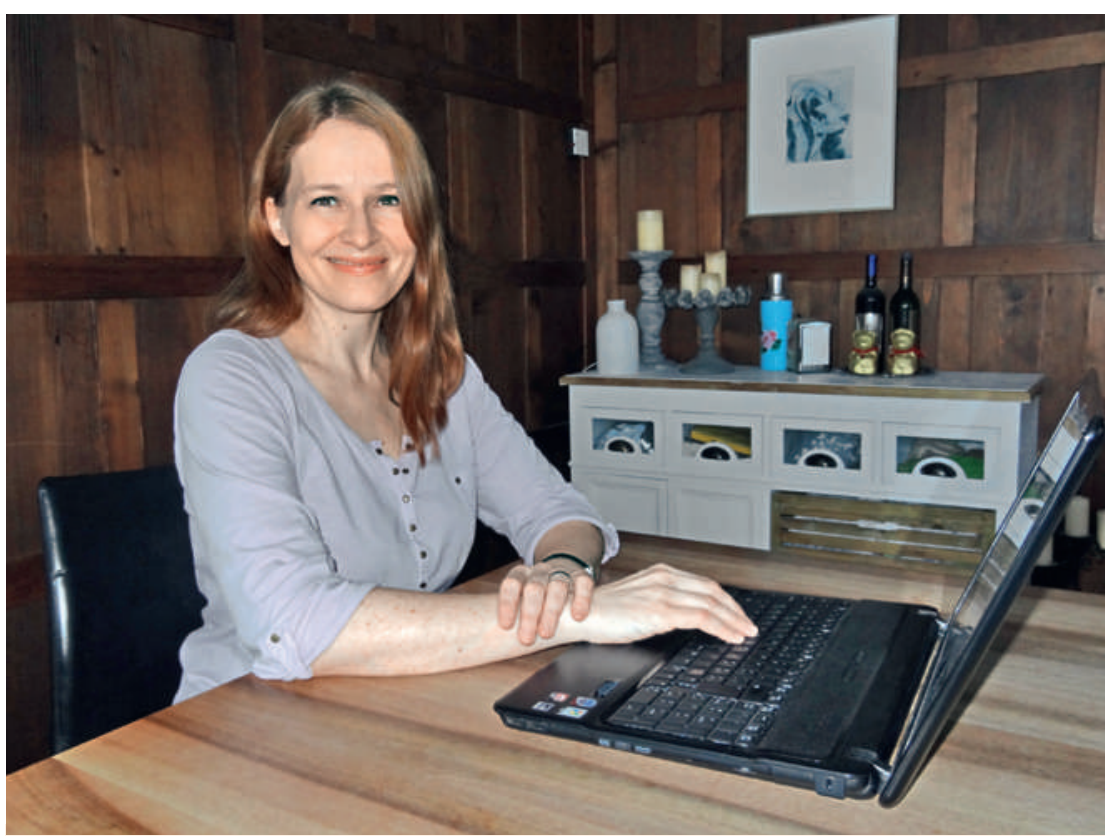

Esther Pauchard: «Bereits bei der Konzeption der Handlung achte ich darauf, ein paar falsche Fährten zu setzen.» ist nicht nur eine Berufskollegin Esther Pauchards, sie ist ebenfalls Mutter von einer, später gar von zwei Töchtern, verheiratet mit einem fürsorglichen Hausarzt und lebt mit ihrer Familie in Thun.

Junge Damen aus zwei Parallelklassen eines Gymnasiums in Romanshorn haben nach der Lektüre von «Jenseits der Couch» gar einen Fanclub zu Ehren von Marc, Kas charmantem Ehemann, gegründet. Esther Pauchards Gatte scheint dem schmeichelhaften Bild seines fiktiven «Doubles» ziemlich genau zu entsprechen, denn schliesslich habe sie tatsächlich «den besten aller Ehemänner», meint die Autorin augenzwinkernd. Auch Kas Pfeife rauchender Vorgesetzter, Bernhard Leutwyler, ähnelt in manchen Charakterzügen jenem Mann, unter dessen Leitung Esther Pauchard einst gewirkt hatte: Die Rede ist von Mario Renz, ärztlicher Direktor am Psychiatriezentrum Münsingen. Er ist ebenso klug, umsichtig und verständnisvoll wie sein leibhaftiges Pendant. Ist Kassandra Bergen demnach das Alter Ego der Autorin? «Ich hatte nie im Sinn, eine autobiographische Geschichte zu schreiben. Auch meine Patienten müssen niemals fürchten, ihren Fall zwischen zwei Buchdeckeln wiederzufinden», stellt sie klar. «Aber dass einem viel mühselige Recherchearbeit erspart bleibt, wenn man eine Kulisse wählt, die einem selbst bestens vertraut ist, stimmt sicher. Ausserdem bietet die Ich-Erzählform unbestreitbare Vorteile, weil sie es erlaubt, die Leserschaft durch die subjektiv gefärbte, in gewisser Weise beschränkte Perspektive der Krimiheldin gezielt in die Irre zu führen.» Jetzt huscht ein spitzbübisches Grinsen über Esther Pauchards Gesicht. «Bereits bei der Konzeption der Handlung achte ich darauf, ein paar falsche Fährten zu setzen.» Wie raffiniert diese gewählt sind, zeigt sich etwa daran, dass sich manchmal nicht nur die Figuren, sondern auch die Leser leichtfertig von ihren Vorurteilen leiten lassen. Gegen Schluss von «Jenseits der Mauern» heisst es bedeutungsvoll: «Kann man einen Menschen jemals richtig einschätzen? Kennen wir überhaupt jemanden wirklich?»

\section{«Menschen wie du und ich»}

Genau solch brisante Fragen sind es, mit denen Esther Pauchard zum Nachdenken anregen möchte. Im Umgang mit psychisch Kranken tendieren viele Menschen dazu, mitunter verworrene Äusserungen voreilig als Krankheitssymptom abzutun. So ergeht es auch Doris Greub, einer Patientin aus «Jenseits der Couch». Später erweist sich jedoch, dass an den Missbrauchsvorwürfen gegen ihren Mann - zunächst als Ausdruck ihrer entgleisten Schizophrenie gewertet - tatsächlich etwas dran ist. «Letztlich können wir nie mit Bestimmtheit sagen, was realen Tatsachen entspricht und was Teil des wahnhaften Erlebens eines Psychiatriepatienten ist. Eine gewisse Unsicherheit bleibt immer.» Esther Pauchard liegt viel daran, dem Laienpublikum einen realitätsge-

\section{Reales Lebensumfeld als Romankulisse}

Wer diese Einrichtung kennt, dem wird schnell klar, dass sie dem fiktiven Arbeitsort von Pauchards Protagonistin Kassandra, «Ka» Bergen - der psychiatrischen Klinik Eschenberg - als Vorbild gedient haben muss. Überhaupt lassen sich zwischen dem fiktionalen Setting und dem realen Lebensumfeld der Autorin einige Affinitäten ausmachen: Kassandra Bergen 
treuen Einblick in den Alltag einer psychiatrischen Klinik zu geben. Dafür eignet sich der fiktionale Rahmen eines Kriminalromans hervorragend, weil er der Leserschaft eine behutsame, unverbindliche Konfrontation mit heiklen, weil oftmals gesellschaftlich tabuisierten Themen ermöglicht. Auf diese Weise können erste Hemmschwellen abgebaut werden. «Ich möchte zeigen, dass psychisch Kranke Menschen sind wie du und ich, mit ihren Problemen, Hoffnungen, Ängsten und Geheimnissen», erklärt sie. Dass dabei die vermeintlich starren Grenzen zwischen Kategorien wie «gut» und «böse» oder «Wahrheit» und «Wahnsinn» verwischen, versteht sich von selbst. manchmal unvernünftige, aber pflichtbewusste und kompetente Kassandra Bergen in gefahrvollen Situationen absichtlich die Regeln des ärztlichen Berufsethos verletzt, um ihre eigene Haut zu retten.

\section{Es bleibt spannend}

Vielleicht verfügt die sympathische, bodenständige Ärztin deshalb über ein so feines Gespür für radikale Perspektivenwechsel, weil sie aufgrund eines angeborenen Hüftleidens in ihrer Kindheit immer wieder Zeit in Krankenhäusern verbracht hatte. Fest steht hingegen, dass es ihr dank ihrer scharfsinnigen, kritischen Beobachtungsgabe gelungen ist, die Leserschaft auf einfühlsame und gleichzeitig höchst amü-

\section{Ihr liegt viel daran, dem Laienpublikum einen realitätsgetreuen Einblick in den Alltag einer psychiatrischen Klinik zu geben.}

So täuscht sich die Protagonistin Kassandra Bergen in Personen, die sie als wertvolle Freunde und Helfer an ihrer Seite zu wissen glaubt. Wenn scheinbar engste Vertraute plötzlich aus dem Lager der Bösewichte heraus agieren, dann ist nicht nur die Krimiheldin geschockt. Freimütig gesteht die Verfasserin, dass sie beim Rätseln auf die falsche Fährte geraten und prompt in die Falle getappt ist. Um die Spannung für zukünftige Leserinnen und Leser zu wahren, soll an dieser Stelle jedoch nicht mehr verraten werden. Im Hinblick auf das soeben erfolgte Bekenntnis allerdings lässt das triumphierende Strahlen in Esther Pauchards Augen keine Zweifel darüber offen, dass sie sehr zufrieden über die Zuverlässigkeit sein muss, mit der ihre bewusst angelegten, kriminalistischen Irrpfade bisweilen beschritten werden. In einer verkehrten Welt wähnt sich der Leser ausserdem, wenn er Zeuge wird, wie die zwar

\footnotetext{
Über die Bücher

Jenseits der Couch (Nydegg Verlag, 2010)

Um halb drei Uhr morgens wird Doris Greub in die psychiatrische Klinik Eschenberg eingeliefert. Verdachtsdiagnose: entgleiste Schizophrenie unter dem Einfluss illegalen Drogenkonsums. Als die Assistenzärztin Kassandra Bergen mit Hilfe der frechen Medizinstudentin Kerstin Lindner beginnt, aus - zunächst medizinischem - Interesse Nachforschungen anzustellen, stossen die Frauen auf viele Ungereimtheiten. Immer tiefer dringen sie in dunkle Geheimnisse vor. Aber die beiden sind nicht die einzigen, die dabei in Gefahr geraten.
}

Jenseits der Mauern (Nydegg Verlag, 2012)

Kassandra Bergen, mittlerweile Oberärztin an der sante Weise in die Welt der Psychiatrie und ihrer Patienten einzuführen. Esther Pauchard nimmt - genau wie ihre Protagonistin Kassandra Bergen - kein Blatt vor den Mund. «Das schönste Kompliment habe ich von einer Frau erhalten, als sie mir mitteilte, sie könne sich nun endlich vorstellen, wie sich ihre an Schizophrenie erkrankte Nichte manchmal fühlen müsse», freut sie sich. Weil sich ihre beiden Kriminalromane so erfolgreich verkauft haben und es der gewieften Autorin teuflischen Spass macht, ihre Figuren auf ihren Abenteuern Allerlei widerfahren zu lassen, hält sie bereits das Konzept zu einem dritten Krimi in der Schublade bereit. «Es könnte sein, dass Marc Hämorrhoiden entwickelt», schmunzelt Esther Pauchard, wobei ein schalkhafter Ausdruck in ihren aufmerksamen Blick tritt. Was wohl seine zahlreichen Verehrerinnen dazu sagen würden? Nicht nur darauf dürfen wir gespannt sein.

psychiatrischen Klinik Eschenberg und überlastet von ihrem enormen Arbeitspensum, ist wenig begeistert, als sie sich auch noch um einen besonders heiklen Fall kümmern soll: Cedric Schwander, ein gefährlicher Gewalttäter, hat einen jungen Mann zu Tode geprügelt. Was mag inn zu dieser grauenhaften Tat getrieben haben? Neugierig tastet sich die unverbesserliche Oberärztin in Schwanders Welt vor. Ohne es zu ahnen, gerät sie dabei ins Visier einer kriminellen Organisation. Die Grenzen zwischen Gut und Böse verwischen, so dass Kassandra Bergen davon absehen muss, ihr medizinisches Wissen lege artis einzusetzen - damit sie überlebt.

(Weitere Informationen: www.esther-pauchard.ch) 